\title{
Spontaneous Bronchopleural Fistula Caused by a Cavitating Rheumatoid Mass
}

CIARAN F. HEALY, MD, MRCS, FFRRCSI; JOHN G. MURRAY, MRCPI, FRCR, FFRRCSI, Department of Diagnostic Radiology, Mater Misericordiae University Hospital, Dublin, Ireland. Address correspondence to Dr. C. Healy, Mater Misericordiae University Hospital, Radiology, Eccles Street, Dublin 7 , Ireland. E-mail: healyciaran@yahoo.com. J Rheumatol 2015;42:137-8; doi:10.3899/jrheum.140408

Pulmonary complications in rheumatoid arthritis (RA) are well documented in the literature and include pleural disease, pulmonary infection, pneumonitis, bronchiolitis obliterans, bronchiectasis, interstitial lung disease, and bronchogenic carcinoma. Rheumatoid pulmonary nodules are uncommon, affecting $<5 \%$ of patients with RA, and are more common in men ${ }^{1}$. They may be solitary or multiple, and are usually located peripherally in the subpleural space. Cavitation is estimated to occur in $<50 \%$ of patients, less commonly than with granulomatosis with polyangiitis (Wegener's)-related pulmonary nodules ${ }^{2}$. Spontaneous pneumothoraxes complicating these nodules have been reported, but they are uncommon. Occasionally, rheumatoid nodules can present as chronic pneumothoraxes of unknown origin. RA may be occult at presentation.

A 43-year-old woman with severe seropositive RA diagnosed at the age of 13 presented to the Emergency Department with worsening shortness of breath. Over a 3-year period prior to this presentation, she was being followed up by both rheumatology and respiratory services for biopsy-proven multiple and bilateral subpleural

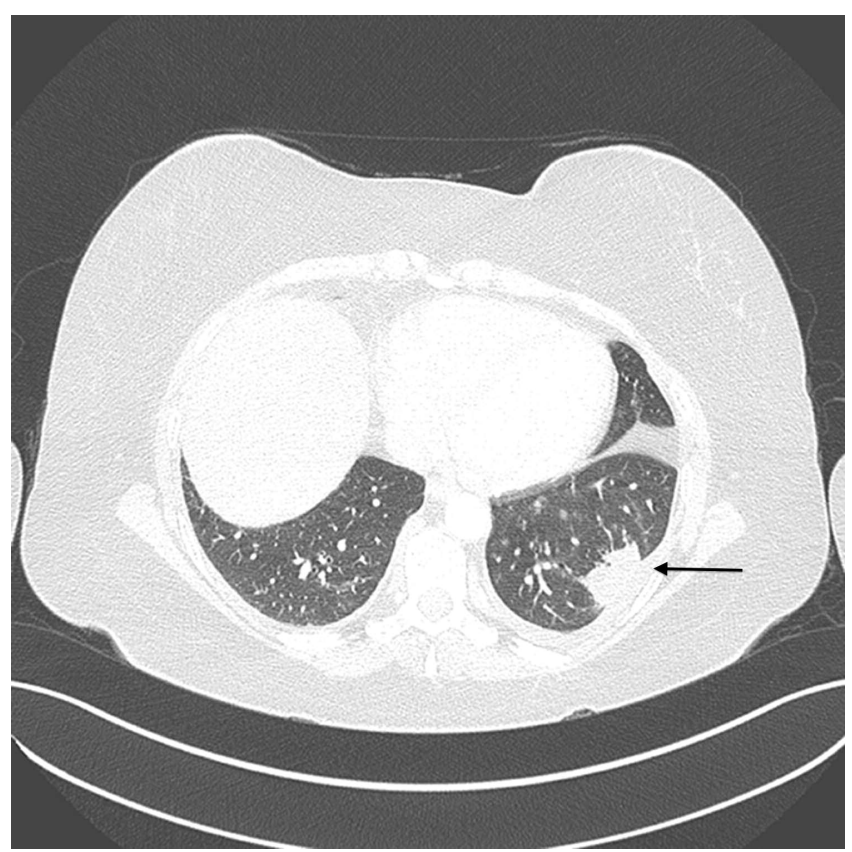

Figure 1. Axial computed tomography of the chest. Noncontrast on lung windows demonstrates a left lower lobe pleural-based noncalcified mass measuring $3.4 \mathrm{~cm}$ in transverse diameter (black arrow).

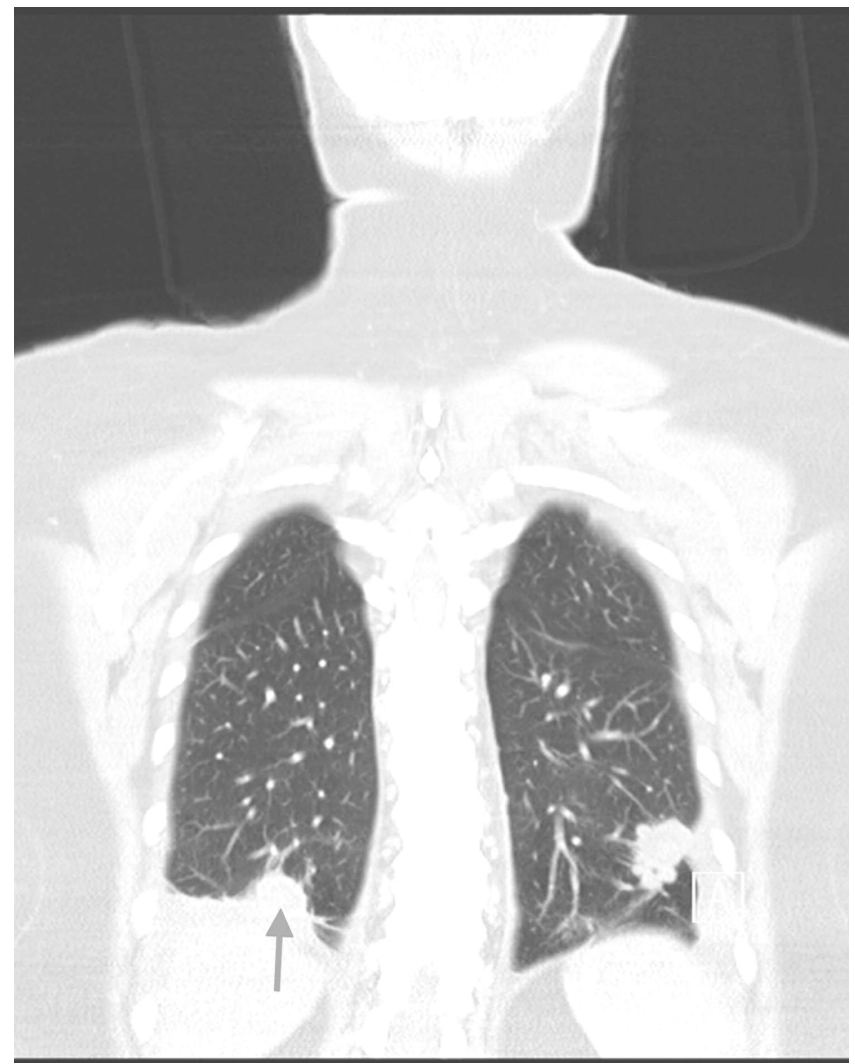

Figure 2. Coronal computed tomography of the chest. Lung windows demonstrating a second pulmonary mass, located peripherally in the right lower lobe in a subpleural location abutting the right hemidiaphragm (gray arrow). Note left lower lobe noncavitating pulmonary mass as depicted in Figure 1.

rheumatoid pulmonary nodules and masses (Figure 1 and Figure 2). Her disease had been managed with a combination of disease-modifying agents over many years. Her most recent combination therapy was methotrexate $15 \mathrm{mg}$ weekly; folic acid, calcium vitamin D, and etoricoxib $90 \mathrm{mg}$ once daily; and prednisolone $5 \mathrm{mg}$ once daily (previously unsuccessful treatment with adalimumab and hydroxychloroquine sulfate). Although she complained of multiple flares, her most debilitating symptoms were tenderness in her proximal interphalangeal joints and shortness of breath.

A computed tomography of the chest, performed during this acute admission, again revealed multiple bilateral pulmonary nodules and masses, one of which demonstrated new cavitation and fistulation with the left pleural cavity, resulting in the formation of a spontaneous bronchopleural

Personal non-commercial use only. The Journal of Rheumatology Copyright @ 2015 . All rights reserved. 


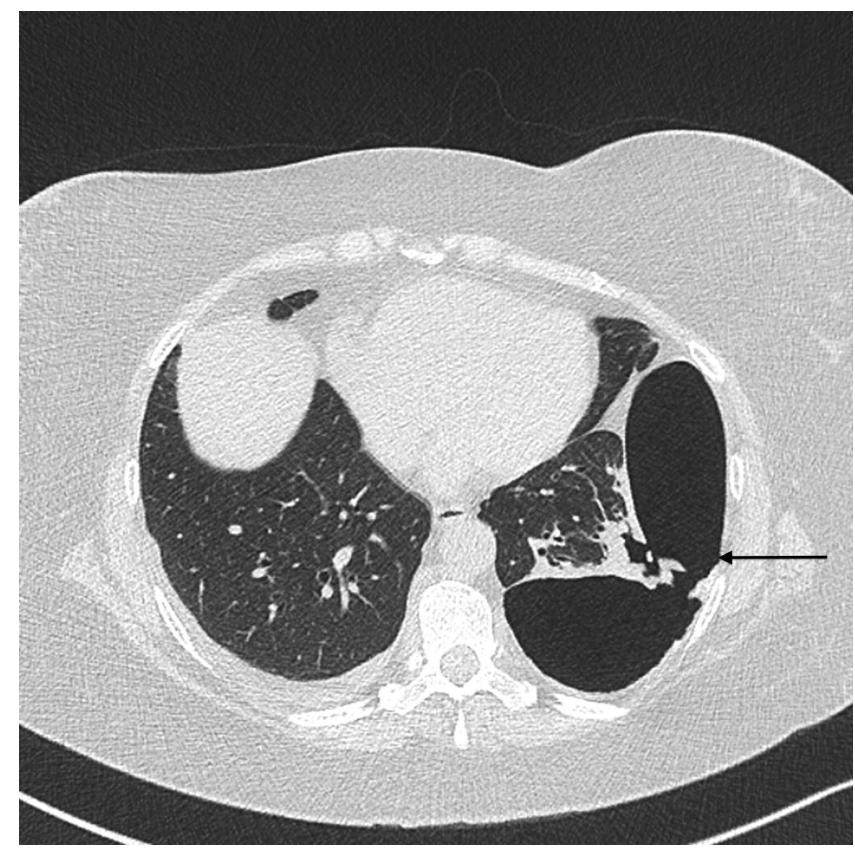

Figure 3. Axial computed tomography of the chest. Noncontrast on lung windows demonstrates a left lower lobe pulmonary mass that has cavitated and formed a spontaneous pneumothorax and bronchopleural fistula (black arrow). fistula (Figure 3 and Figure 4). This was initially treated with chest drain insertion, resulting in symptomatic relief, followed by successful definitive management with direct surgical repair.

\section{REFERENCES}

1. Terasaki H, Fulimoto K, Hayabuchi N, Ogoh Y, Fukuda T, Muller NL. Respiratory symptoms in rheumatoid arthritis: relation between high-resolution CT findings and functional impairment. Radiat Med 2004;22:179-85.

2. Gadkowski B, Stout JE. Cavitary pulmonary disease. Clin Microbiol Rev 2008;21:305-33.

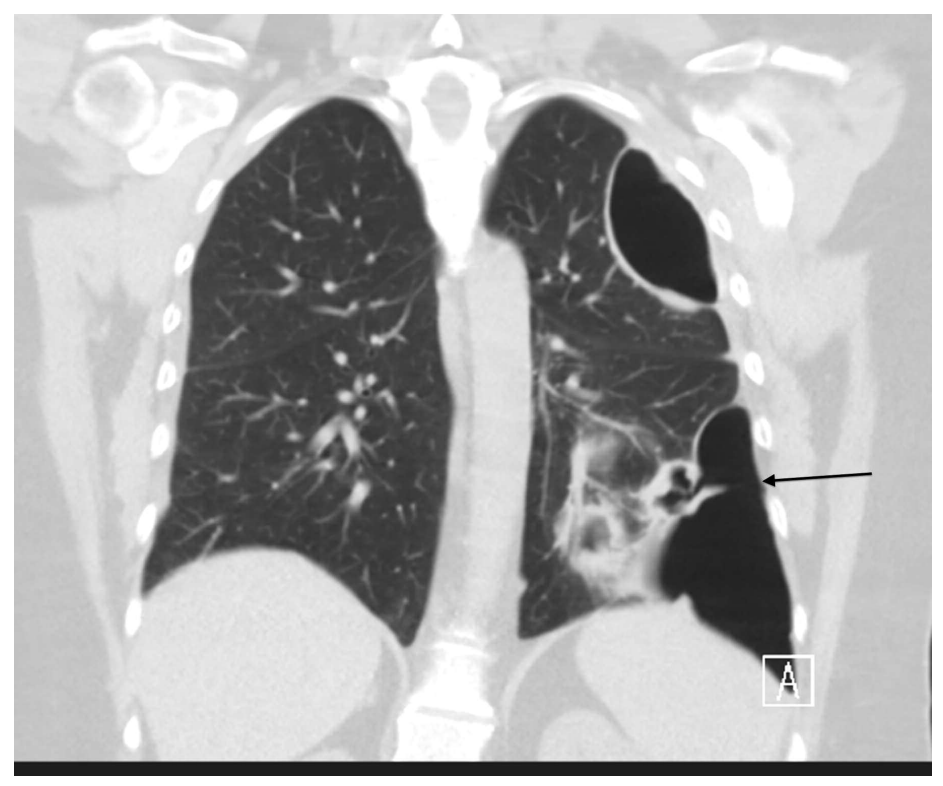

Figure 4. Coronal reconstruction computed tomography of the chest. Lung window demonstrates the left lower lobe pulmonary mass that has cavitated and formed a spontaneous pneumothorax and a bronchopleural fistula (black arrow). 